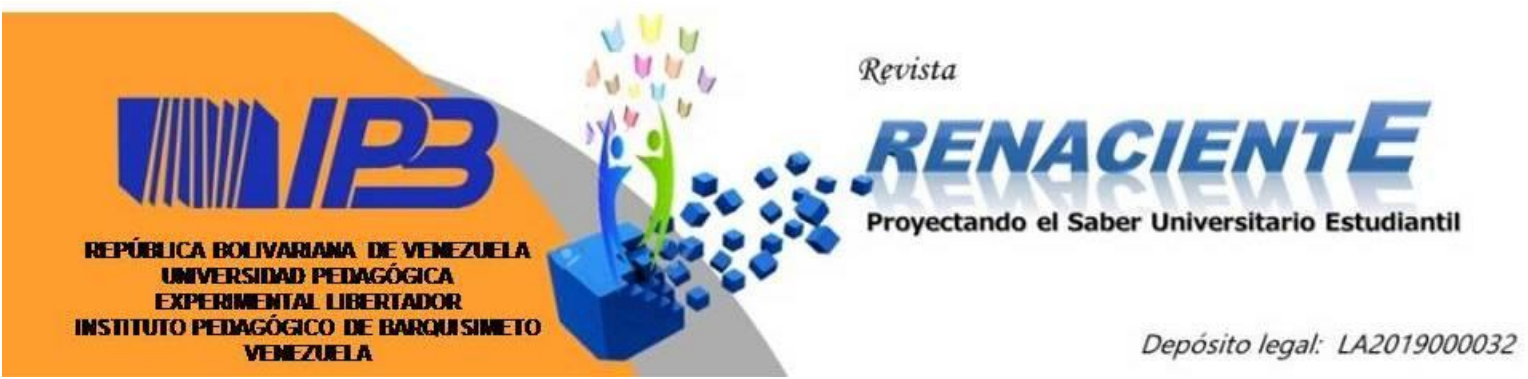

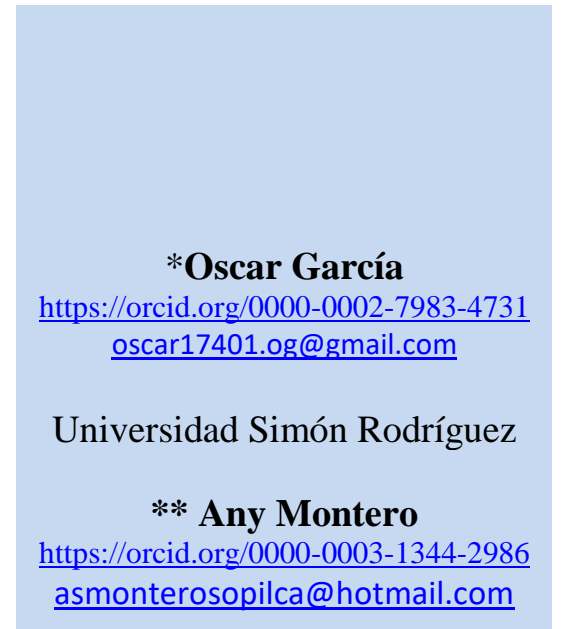

Universidad Pedagógica

Experimental Libertador. Instituto Pedagógico de Barquisimeto (UPEL IPB) Venezuela.

* Estudiante de Administración. Mención Mercadeo de la Universidad Simón Rodríguez, Barquisimeto. Estado Lara

**Docente y Tutora de la UPELIPB, Barquisimeto. Estado Lara.

***El artículo presentado apoya a una investigación libre vinculada a la Línea de Investigación Haceres y Saberes de la Investigación Educativa, del Núcleo de Investigación NIDIT UPEL IPB

\section{ESTADO EMOCIONAL DE ESTUDIANTES DEL COLEGIO "PABLO VI" EN EL CONTEXTO SOCIAL VENEZOLANO Y SU RELACIÓN CON LOS PROCESOS COGNITIVOS****}

\section{Resumen}

Esta investigación, pretende determinar el grado de relación entre el estado emocional del estudiante de 1er Año del Colegio "Pablo VI" y los procesos cognitivos que éste desarrolla. Se desarrolla estudio de tipo descriptivo correlacional, se utilizaron dos test estandarizados como instrumentos. Las variables fueron descritas y relacionadas en el contexto social-económico de Venezuela, existe la intención de generar conocimiento que ayude en la resolución de la problemática que subyace en el estudio, proponiendo recomendaciones a docentes y directivos en pro del bienestar estudiantil. Fueron utilizadas bases teóricas que sustentan las ideas y posibles escenarios en estudio. Fue considerada una muestra de 11 estudiantes a quienes se les aplicó los test, obteniendo los resultados que fueron tabulados y graficados a través de la estadística descriptiva y utilizando el coeficiente de correlación de Spearman, los resultados permitieron tener una visión de que existe una alta relación entre las variables estudiadas.

Descriptores: Estado emocional, Procesos cognitivos,Bachillerato

\section{STUDENTS OF THE SCHOOL "PABLO VI" IN THE VENEZUELAN SOCIAL CONTEXT AND ITS RELATIONSHIP WITH COGNITIVE PROCESSES Abstract}

This investigation research to determine the degree of relationship between the emotional state of the 1st Year student of the School "Pablo VI" and the cognitive processes that it develops. Study of correlational descriptive type is developed; two standardized tests were used as instruments. The variables were described and related in the social-economic context of Venezuela, there is the intention to generate knowledge that helps in the resolution of the problem underlying the study, proposing recommendations to teachers and managers for student well-being. Theoretical bases were used to support ideas and possible scenarios under study. It was considered a sample of 11 students who were tested, obtaining the results that were tabulated and plotted through descriptive statistics and using Spearman's correlation coefficient, the results allowed to have and view that there is a high relationship between the variables studied.

Key words: Emotional state, Cognitive processes, high school 


\section{Introducción}

El individuo es un ser social que busca la inserción en la colectividad para ser un ente activo y productivo de la misma; y todo ello, es alcanzado a través de la educación, pues según afirma Rivas (2015) ha sido considerada como un elemento clave para el desarrollo humano y es una pieza fundamental para la sustentabilidad; ya que a través de ésta cada quien se hace consciente del impacto de sus actividades sobre el entorno.

Adicionalmente, a través de la formación el individuo, quien tiene características complejas y multidimensionales, puede conseguir un equilibrio, conjugar armónicamente lo biológico, lo psicológico y lo social. De tal forma que, cuando uno de estos elementos se encuentra en desequilibrio ocurren conflictos en cualquiera de los otros elementos del individuo, debido a que el todo está interconectado como un sistema que coadyuva en el desenvolvimiento adecuado del ser.

Las emociones que representan un componente psíquico, ejercen una amplia influencia sobre la personalidad; también son factores significativos en la determinación de actitudes y representan un papel primordial en las relaciones del individuo con los demás. Una gran parte del valor y significado de la vida se centra en las relaciones emocionales y adecuadas, por consiguiente, la falta de control emocional constituye un factor básico causal de conflicto en casi todas las formas de desarrollo cognitivo.

En consecuencia a esta idea, Muñoz (2019) refiere que muchas de las personas que presentan un equilibrio psicológico y emocional suelen tener un enfoque realista de la vida, no se ven afectadas con facilidad por los problemas o reveses cotidianos. Por eso, ante situaciones de estrés o dificultades, reaccionan con moderación y suelen reponerse con una relativa facilidad. Sus emociones son estables, no se dejan llevar por ellas, tampoco por sus impulsos, en otras palabras, no presentan grandes cambios de humor, ni de ánimo, son pacientes, tranquilas, perseverantes y confiables.

Las personas con una alta estabilidad emocional funcionan de una manera flexible y a la vez controlada, a la vez que son capaces de dar mejor respuesta en su aprendizaje. Por otra parte, Lozano (2019), señala que en las instituciones persisten las clases monótonas con recursos pasivos donde el educando recibe la información teniendo que desarrollar un aprendizaje memorístico, repetitivo, poco inventivo y participativo. Por lo cual difiere con

89 Oscar García y Any Montero: Estado emocional de estudiantes del colegio "Pablo VI" en el contexto social venezolano y su relación con los procesos cognitivos. Pp.88-109 
que los docentes se enfilen sólo en las actitudes superficiales, sin actividad crítica y participativa, dejando en lo más mínimo el desarrollo emocional del mismo. Por lo tanto, se requiere un ajuste curricular en educación el cual contemple la acción complementaria del ser.

Esta problemática descrita, es percibida en un contexto significativo para los jóvenes venezolano quienes experimentan en la actualidad una crisis general a nivel social que afecta indiscutiblemente a su ser, además, de la difícil situación que afronta este país en la actualidad en los ámbitos políticos, económicos y sociales, los cuales en consecuencia afectan prolongadamente a cada sujeto y más aún a los estudiantes en formación.

Desde esta perspectiva, se puede ver reflejada esta situación en cada hogar venezolano, ya que se ha producido la escasez de alimentos, medicinas, insumos diarios y productos básicos; a la vez que, se enfrenta una galopante inflación, todo ello dado por el estancamiento de la producción general que existe en el país hoy en día, lo que obliga a buscar a padres a buscar alternativas de sustentabilidad que en ocasiones provoca desajustes emocionales y estrés.

En este sentido Revilla (2010), plantea que el escenario del caos con indignación ciudadana activa, explica que el resultado de algo depende de distintas variables y que es imposible predecir, el futuro no está escrito en ninguna parte. Sin embargo, se pueden construir algunos escenarios, muy a pesar de estar hoy inmersos en un entorno político de incertidumbre y de estancamiento económico, en este escenario del caos se puede desatar una conflictividad mayor, si se renuncia al dialogo, a la negociación y a la mediación como herramientas básicas de la acción política y si se sigue apostando a la confrontación y sin proyecto país viable y sostenible, la crisis institucional será cada día más profunda y todos los sectores sociales, incluida el núcleo familiar seguirán pagando a alto costo la falta de entendimiento e incomprensión de los actores predominantes en la sociedad venezolana actual.

Por tanto cada vez más se hace evidente la preocupación, el estrés y la desilusión que se conoce actualmente en el venezolano, ya que no se garantizan derechos humanos básicos como la alimentación, transporte, salud y seguridad, por lo cual, parte de la población se encuentra en un descontento que los lleva a hacer prácticas inusuales en su 
rutina diaria, además, la insuficiencia monetaria que perciben los trabajadores venezolanos conlleva a que tengan que recurrir a trabajos extras para poder sustentarse económicamente, y a pesar de esto no logran cubrir sus necesidades básicas.

Según Canelones (2016), la crisis en Venezuela cumple con las tres condiciones necesarias que definen al estrés crónico y agudo: son situaciones materiales, psicológicas o sociales nuevas; son evaluadas por las personas como amenazantes o desbordantes de los recursos individuales para solucionarlos $\mathrm{y}$, por último, ponen en peligro su bienestar psicofísico y social. Es por ello que la hostilidad, la irritabilidad, la frustración y la ansiedad no solo son evidentes en la calle, sino también en el trabajo, en el hogar y en el colegio; por otra parte, esta situación que persigue a los venezolanos produce un cierto tipo de estrés el cual se puede convertir en crónico y genera agotamiento físico y mental e igualmente se puede complicar con un cuadro depresivo que requiere atención especializada y que a su vez repercute en la inestabilidad de los otros componentes y sistemas del individuo incluso el elemento cognitivo.

En correspondencia a este panorama descrito, es intención de la presente investigación determinar si existe una relación entre las variables en estudio, teniendo como variable interviniente el contexto social venezolano.

\section{Apoyo Teórico}

La dinámica cambiante de la sociedad actual plantea nuevos retos a la educación que debe ser asumido a la brevedad posible; distintos grupos de personas se han dedicado con el paso de los años a estudiar y analizar los procesos que se propagan dentro de nuevas teorías desarrolladas al pasar de los años. Dentro de este marco se realzan diversos conceptos fundamentales que permitirán explicar y dar sentido a los distintos procesos en el contexto estudiado.

\section{Estado Emocional}

Una emoción es un estado afectivo que se experimenta, una reacción subjetiva al ambiente que viene acompañada de cambios orgánicos, estos tienen una función adaptativa del organismo al entorno, Fernández-Abascal y cols. (2010). De esta manera, se puede señalar que el mismo es un estado que sobreviene bruscamente en forma de crisis 
relativamente rápida. Asimismo, es importante mencionar que en el ser humano la experiencia de una emoción generalmente involucra un conjunto de cogniciones, actitudes y creencias sobre el mundo, que son utilizados para valorar y adaptarse a una situación concreta, por tanto, influyen en el modo de percibir distintas situaciones.

De esta manera, resulta oportuno advertir que las emociones son un todo resultante e interactuante de los múltiples sistemas que conforman el todo complejo de los individuos y que llevados de forma coherente permiten un desarrollo equilibrado de los seres humanos. De tal forma, que al individuo al estar en armonía con los sistemas biológicos, psicológicos y relacionales tendrá un mejor desarrollo de su ser (Maturana en Ocaña, 2015).

Por otra parte, cabe señalar que durante mucho tiempo las emociones han sido consideradas poco importantes y se le ha otorgado más relevancia al sentido racional y cognitivo del individuo, dejando pasar desapercibido que las emociones al ser estados afectivos, indican estados internos personales, motivacionales, de necesidades y objetivas que coadyuvan al desarrollo racional y personal de cada ser ya que en correspondencia a su personalidad, cada individuo podrá responder de formas indistintas, es difícil predecir la acción ante el hecho.

En tal sentido, cada individuo experimenta una emoción de forma particular, dependiendo de experiencias anteriores, aprendizajes, carácter, y de la situación concreta. En este punto se hace perentorio, mencionar a Goleman (1990), quienes expresaron la necesidad de resaltar la inteligencia emocional para explicar o comprender la acción emocional en la cognición del ser y aun cuando esta no se presenta como una cualidad especial que solo tengan unos pocos, ponen de relieve la necesidad de prestar atención a este concepto que puede significar un gran avance en materia racional al momento de manejar correctamente las emociones.

\section{Procesos Cognitivos en los adolescentes}

La capacidad de desarrollar conocimientos recibe el nombre de cognición, se trata de la habilidad para procesar y asimilar datos a partir de la experiencia, percepción u otros. Según Villalta et.al. (2013), los procesos cognitivos son procedimientos que lleva a cabo el ser humano para incorporar conocimientos, en los cuales intervienen facultades como la 
inteligencia, la atención, la memoria y el lenguaje.

Por ende, los procesos cognitivos pueden analizarse desde diferentes ciencias y disciplinas. Es importante decir, que el pensamiento también juega un papel fundamental dentro del proceso cognitivo, procesa toda la información y luego establece relaciones entre los datos que los componen, mediante acciones como el análisis, el razonamiento, la asimilación, la síntesis y la resolución de problemas.

Debido a esto se observa que los procesos cognitivos se pueden ver afectados por los estados emocionales de las personas ya que este participa o juega como un papel principal dentro de la motivación, desempeño y esfuerzo del individuo. Asimismo, existen amplios debates en torno a los procesos cognitivos, estos pueden realizarse de forma consciente o inconsciente e incluso, hasta pueden ser desarrollado por animales o por entidades construidas por el hombre.

En vista de la situación planteada, un proceso cognitivo puede iniciarse con una percepción, la persona atiende a aquello que percibe y a través de distintos tipos de pensamientos y mecanismos de inteligencia, logra generar un conocimiento que interioriza y almacena dentro de sí. Además, un proceso cognitivo implica una cierta información codificada por el individuo, para que cada vez que una situación lo amerite, el sujeto pueda expresar dicha información y utilizarla con respecto a su necesidad.

En este orden de ideas, existen diversas teorías del aprendizaje como lo afirma Arranz (2017) que, exceptuando algunos defensores del aprendizaje asociativo, ninguna ignora los procesos mentales y afirma que además ningún proceso cognitivo en el desarrollo del conocimiento suele actuar de forma independiente. El individuo se esfuerza por integrar todos los recursos para mejorar los hábitos de estudio y lograr un aprendizaje significativo.

Por su parte la revista ABC de la psicología (2012), en su artículo el desarrollo cognoscitivo de los adolescentes, diserta que, de acuerdo a Piaget, las operaciones formales ocurren entre los 11 años en adelante, y corresponden a la etapa de la pubertad y adolescencia en adelante. Así, la mencionada etapa de las operaciones formales, es el estadio (fase) final del desarrollo cognoscitivo, que se caracteriza por el pensamiento abstracto y se genera el proceso formal del pensamiento. 
Según los autores señalan que los estudios de este psicólogo, resaltan que el estadio de las operaciones formales es el punto más alto que cualitativamente alcanza el ser humano en su desarrollo intelectual y una vez es alcanzada, todos los progresos que se realizan son llevados de forma más cuantitativos. Es necesario señalar que, en la adolescencia, el pensamiento cambia, considerablemente y que la fase operacional de los jóvenes se le conoce también como la etapa de la conquista del pensamiento.

En suma, los adolescentes en la etapa de las operaciones formales según Piaget tienen la capacidad de razonar y deducen conclusiones a partir de conceptos abstractos. Poco a apoco desde la pubertad, el adolescente revisa y analiza sus creencias y modifica su visión de las cosas y del mundo, los mismo experimentan una flexibilización del pensamiento, es decir, palabras o frases que manejaban antes con rigurosidad de conceptos pueden tener para ellos, una amplia gama de significados, que, en la etapa de la niñez, los símbolos sólo pueden tener sentido si se les explica con palabras, si es algo idea concreto y comprobable.

En tal sentido, afirma la revista $\mathrm{ABC}$ que el adolescente puede construir múltiples interpretaciones de una situación que observa, es decir, no tiene una sola respuesta, sino varias; y comprender alternativas ante resultados inesperados, por tanto, reconoce que algunas situaciones no tienen respuestas definitivas. Asimismo, en la etapa operacional formal, los adolescentes tienen la capacidad de orientarse hacia lo abstracto y lo que no está presente. Esto le brinda la posibilidad de un pensamiento en el futuro, la interpretación de la diferencia entre lo real y lo posible y la posibilidad de recrear y generar nuevos pensamientos, que lo convierten en un ser más inventivo, imaginativo, original y la posibilidad domina la realidad.

En conclusión, los procesos cognitivos permiten reconocer de forma coherente distintas informaciones que conllevan a distintos procesos, con el fin de estimular la mente y así desarrollar una madurez cognitiva, aunado a esto, los procesos mentales en psicología son analizados con el fin de ayudar a mejorar la calidad de vida, puesto que es elemental que aprendamos a desarrollarlos y gestionarlos desde el momento del nacimiento. 


\section{Orientación Metodológica}

La naturaleza de la investigación de este trabajo se orienta por el paradigma positivista siguiendo un enfoque cuantitativo, el cual se basa en fenómenos observables que son susceptibles a medición, análisis matemático y control experimental. En este mismo sentido, Hernández et.al. (2014), señalan que las investigaciones de orden cuantitativo están dirigidas a medir fenómenos, comparar resultados e interpretarlos en función de la teoría referencial que se posee, tomando en cuenta las variables que pudiesen haber influido.

Adicionalmente, las investigación es de tipo correlacional según Hernández et.al. (2014), son consideradas una modalidad independiente de los estudios descriptivos. En este sentido, estas son una serie de investigaciones que describen un fenómeno, para determinar el grado de asociación existente entre las variables. Para Arias (2003) la finalidad de estos estudios es encontrar la relación (no causal) entre dos o más variantes. Principalmente, se miden las variables y posteriormente mediante pruebas de hipótesis correlacionales y la aplicación de técnicas de estadísticas, se estima la correlación.

\section{Diseño de la Investigación}

El diseño de investigación se refiere a la estrategia general que asume el investigador para obtener solución al problema planteado. Además, constituye la estructura de cualquier investigación científica, así mismo brinda dirección y procede a sistematizar los estudios (Arias, 2003). De este modo, siendo este estudio una investigación descriptiva de tipo correlación al, apoyada en una investigación de campo, es necesario señalar que es aquella que se encarga de la recolección de datos principalmente de los individuos analizados, o del ámbito donde son ocurridos los hechos (datos primarios).

\section{Técnicas e Instrumentos de Recolección de la Información}

Las técnicas, son procedimientos que favorecen la adquisición de la información pertinente y oportuna, además de significar un aporte para recolección de forma concreta y rápida al aplicar la encuesta de forma directa. Al respecto, Sabino (2002) "si se quiere conocer algo sobre el comportamiento de las personas, lo mejor, más directo y simple es preguntárselos directamente a ellos" (p.45).

95 Oscar García y Any Montero: Estado emocional de estudiantes del colegio "Pablo VI" en el contexto social venezolano y su relación con los procesos cognitivos. Pp.88-109 
En relación con lo anterior, se establece entonces como técnica para este trabajo la encuesta, la cual es definida por Ruíz Bolívar (2013) como una "estrategia utilizada para la recogida de datos, antecedentes e información, lo cual facilita no solamente la medición de las variables, sino que dimensiona el escenario posible para llegar a resultados u opiniones emitidos por los usuarios”.(p. 56)

Asimismo, el instrumento que se pretende utilizar es el cuestionario, definido por Ruíz Bolívar (2013) como: "un medio de comunicación escrito entre el encuestador y el encuestado, facilite traducir los objetivos y las variables del hecho investigado mediante un conjunto de preguntas muy particulares previamente preparadas, a fin de fortalecer la investigación" (p. 78).

Es importante mencionar, que para la presente investigación serán estudiadas dos variables las cuales serán relacionadas, la primera es la estabilidad emocional del estudiante del primer año del colegio Pablo VI y la segunda el alcance de los procesos básicos cognitivos, percibidos a través del rendimiento académico.

Para la primera de las variables se hará uso de un test estandarizado debidamente validado, el mismo fue realizado por Belles (2010) y fue sometido a todos los estudios técnicos de rigor y necesarios para poder ser usado. La autora en su estudio determina que el instrumento es apto, fiable y valido. El mismo consta de 23 ítems que satisfacen las dimensiones en estudio y ofrece un cuadro que permite la disertación del índice alcanzado.

Mientras que para el rendimiento académico será tomado en cuenta el índice académico de la muestra en cuestión a través de un registro de las calificaciones obtenidas en lo que va de año escolar.

\section{Técnicas de Análisis e Interpretación de la Información}

Mediante el análisis de los datos recolectados se busca obtener la relación existente entre las dos variables sometidas al estudio. Los cuáles serán tabulados, codificados y procesados por medio del estadístico de correlación de Spearman, el cual, según Herrera y Montero (2013) es una prueba estadística que permite establecer el grado de relación positiva o negativa existente entre dos variables en escalas ordinal.

Para determinar el grado de relación existente entre las variables se utilizará la 
fórmula propuesta por Spearman ya que disco estadístico trata de medir la asociación de dos variables discretas, en un nivel de medición ordinal, en su magnitud. Se le conoce como correlación a través de Rangos (r). La correlación de Spearman, es un valor que se obtiene mediante la fórmula:

Es importante señalar que, por tratarse de estudio descriptivo, previo al establecimiento de la relación se realizó un estudio descriptivo haciendo uno de tablas y gráficos de las dimensiones del instrumento aplicado a los estudiantes.

\section{Desarrollo e Interpretación de los Resultados}

Para efectos del procesamiento de los mismos, se procedió a tomar una muestra de individuos, quienes fueron encuestados bajo dos instrumentos en relación con las variables en estudio. Los mismos tienen como finalidad determinar el dominio de los procesos cognitivos y, el estado emocional que cada individuo experimenta en su entorno.

Una vez determinados los resultados de manera individual en ambos instrumentos de emocionalidad y procesos cognitivos se detallaron el grado de relación existente entre las dos variables, para tal efecto se realizó el debido análisis a los datos que previamente han sido agrupados según categorías y dimensiones de forma general según la percepción de los mismos.

El proceso se realizó a través del análisis cuantitativo descriptivo y cualitativo interpretativo de la información y la graficación de la misma, asimismo se realiza el análisis individual de los ítems para estructurar conclusiones integrales.

\section{Resultado del Dominio de los Procesos Cognitivos}

Para realizar este apartado, y poder determinar los resultados de los procesos cognitivos de los individuos, principalmente se construyó la siguiente tabla:

Cuadro 1. Tabla referencial para la interpretación del instrumento 1

\begin{tabular}{cc}
\hline Puntajes & $\begin{array}{c}\text { Dominio de los } \\
\text { Procesos Cognitivos }\end{array}$ \\
\hline $\mathbf{1 - 5}$ & Muy Poco Dominio
\end{tabular}

97 Oscar García y Any Montero: Estado emocional de estudiantes del colegio "Pablo VI" en el contexto social venezolano y su relación con los procesos cognitivos. Pp.88-109 


\begin{tabular}{cc}
\hline $\mathbf{6}-\mathbf{1 0}$ & Poco Dominio \\
\hline $\mathbf{1 1}-\mathbf{1 5}$ & Buen Dominio \\
\hline $\mathbf{1 6}-\mathbf{2 0}$ & Excelente Dominio
\end{tabular}

Posteriormente, fue aplicado el instrumento ya estandarizado con el propósito de conocer el dominio en los procesos cognitivos en la muestra seleccionada, se obtuvo el siguiente resultado:

Cuadro 2. Frecuencias de puntajes alcanzados por la muestra

\begin{tabular}{ccc}
\hline $\begin{array}{c}\text { Puntajes } \\
\text { Alcanzados }\end{array}$ & Frecuencia & Porcentaje (\%) \\
\hline $\mathbf{1 ~ - 5}$ & 1 & $9 \%$ \\
\hline $\mathbf{6}-\mathbf{1 0}$ & 5 & $46 \%$ \\
\hline $\mathbf{1 1}-\mathbf{1 5}$ & 4 & $36 \%$ \\
\hline $\mathbf{1 6}-\mathbf{2 0}$ & 1 & $9 \%$ \\
\hline
\end{tabular}

En correspondencia a los resultados relativos al estudio del dominio de los procesos cognitivos, se encuentra que el $9 \%$ de la muestra tiene muy poco dominio, luego surge un $46 \%$ de la misma que tiene poco dominio. Frente a los mencionados porcentajes se refleja un $36 \%$ que tiene buen dominio y finalmente un $9 \%$ que posee un excelente dominio del estudio planteado. Es importante señalar en este apartado que la cognición es la capacidad del individuo de desarrollar conocimientos, por tanto, se trata de la habilidad para procesar y asimilar datos a partir de la experiencia, percepción u otros. Según Porto (2013), los procesos cognitivos son procedimientos que lleva a cabo el ser humano para incorporar conocimientos, en los cuales intervienen facultades como la inteligencia, la atención, la memoria y el lenguaje.

\section{Resultados del instrumento para determinar el Estado Emocional del individuo}

Por otra parte, se estableció un segundo instrumento estandarizado, el cual fue categorizado en dimensiones para así poder demostrar las distintas percepciones:

Cuadro 3. Tabla referencial para la interpretación de la estabilidad emocional en la Ansiedad 


\begin{tabular}{|c|c|c|c|c|c|c|c|c|c|c|c|c|c|}
\hline \multirow[t]{2}{*}{ Ítem } & \multirow[t]{2}{*}{ Proposición } & \multicolumn{2}{|c|}{ Nunca } & \multicolumn{2}{|c|}{ Casi Nunca } & \multicolumn{2}{|c|}{$\begin{array}{l}\text { Algunas } \\
\text { Veces }\end{array}$} & \multicolumn{2}{|c|}{$\begin{array}{l}\text { Con } \\
\text { Frecuencia }\end{array}$} & \multicolumn{2}{|c|}{$\begin{array}{l}\text { Casi } \\
\text { Siempre }\end{array}$} & \multicolumn{2}{|c|}{ Siempre } \\
\hline & & $\mathrm{f}$ & $\%$ & f & $\%$ & $\mathrm{f}$ & $\%$ & $\mathrm{f}$ & $\%$ & $\mathrm{f}$ & $\%$ & $\mathrm{f}$ & $\%$ \\
\hline 3 & $\begin{array}{l}\text { Pienso que las } \\
\text { personas tienen } \\
\text { malas intenciones y } \\
\text { rara vez dicen la } \\
\text { verdad. }\end{array}$ & 1 & 9 & 2 & 18 & 5 & 46 & 2 & 18 & 1 & 9 & 0 & 0 \\
\hline 4 & $\begin{array}{l}\text { Cuando alguien me } \\
\text { cuenta algo íntimo, } \\
\text { me siento incómodo } \\
\text { y no sé qué } \\
\text { responder. }\end{array}$ & 2 & 18 & 4 & 36 & 3 & 27 & 0 & 0 & 2 & 18 & 0 & 0 \\
\hline 10 & $\begin{array}{l}\text { A veces sueño } \\
\text { despierto, o me } \\
\text { encuentro a mí } \\
\text { mismo con la mente } \\
\text { en blanco, } \\
\text { totalmente relajado, } \\
\text { sin pensar en nada } \\
\text { en concreto. }\end{array}$ & 3 & 27 & 1 & 9 & 2 & 18 & 3 & 27 & 1 & 9 & 1 & 9 \\
\hline 15 & $\begin{array}{l}\text { Suelo analizarme a } \\
\text { mí mismo, pensando } \\
\text { que podría haber } \\
\text { hecho mejor ciertas } \\
\text { cosas. }\end{array}$ & 0 & 0 & 2 & 18 & 3 & 27 & 0 & 0 & 4 & 36 & 2 & 18 \\
\hline 19 & $\begin{array}{lr}\text { Me } & \text { siguen } \\
\text { preocupando ras } \\
\text { cosas que ya han } \\
\text { ocurrido. }\end{array}$ & 0 & 0 & 4 & 36 & 1 & 9 & 3 & 27 & 2 & 18 & 1 & 9 \\
\hline 22 & $\begin{array}{l}\text { Tiendo a repetirme } \\
\text { a mí mismo "no } \\
\text { puedo conseguirlo" } \\
\text { Cuandor estoy } \\
\text { realizando algo } \\
\text { importante. }\end{array}$ & 2 & 18 & 4 & 36 & 2 & 18 & 0 & 0 & 1 & 9 & 2 & 18 \\
\hline & $\begin{array}{l}\text { Puntajes } \\
\text { por opción }\end{array}$ & 33 & & 29 & & 31 & & 4 & & 39 & & $\begin{array}{l}3 \\
3\end{array}$ & \\
\hline & $\begin{array}{l}\text { Media de la } \\
\text { Percepción global de } \\
\text { la muestra }\end{array}$ & $\mathrm{Pt} \mathrm{n}$ & & & & & & 9 & & & & & tmax \\
\hline
\end{tabular}

En el cuadro 3 puede apreciarse en la media de la percepción global de la muestra para la dimensión ansiedad que el resultado apunta a un valor promedio de 19 puntos, lo cual quiere decir que los estudiantes según Bellés (2010) muestran un grado de ansiedad medio, situación que repercute en los índices de tensión y preocupación de dichos sujetos.

Asimismo, se desarrolla la segunda dimensión expuesta en este estudio: 
Cuadro 4. Tabla referencial para la interpretación de la estabilidad emocional en la habilidad para expresar sentimientos

\begin{tabular}{|c|c|c|c|c|c|c|c|c|c|c|c|c|c|}
\hline \multirow{3}{*}{$\begin{array}{l}\text { Ítem } \\
6\end{array}$} & \multirow{3}{*}{$\begin{array}{l}\text { Proposición } \\
\text { Me resulta difícil } \\
\text { expresar una } \\
\text { opinión distinta a } \\
\text { la de la persona } \\
\text { con la que estoy } \\
\text { hablando. }\end{array}$} & \multicolumn{2}{|c|}{ Nunca } & \multicolumn{2}{|c|}{$\begin{array}{l}\text { Casi } \\
\text { Nunca }\end{array}$} & \multicolumn{2}{|c|}{$\begin{array}{l}\text { Algunas } \\
\text { Veces }\end{array}$} & \multicolumn{2}{|c|}{$\begin{array}{l}\text { Con } \\
\text { Frecuenci } \\
\text { a }\end{array}$} & \multicolumn{2}{|c|}{$\begin{array}{l}\text { Casi } \\
\text { Siempre }\end{array}$} & \multicolumn{2}{|c|}{$\begin{array}{l}\text { Siempr } \\
\text { e }\end{array}$} \\
\hline & & $\mathrm{f}$ & $\%$ & $\mathrm{f}$ & $\%$ & $\mathrm{f}$ & $\%$ & $\mathrm{~F}$ & $\%$ & $\mathrm{f}$ & $\%$ & $\mathrm{f}$ & $\%$ \\
\hline & & 2 & 18 & 4 & 36 & 3 & 27 & 2 & 18 & 0 & 0 & 0 & 0 \\
\hline 9 & $\begin{array}{l}\text { Cuando me siento } \\
\text { asustado por algo } \\
\text { importante que va } \\
\text { a tener lugar en mi } \\
\text { vida, me cuesta } \\
\text { admitirlo y no sé } \\
\text { cómo pedir que me } \\
\text { comprendan. }\end{array}$ & 0 & 0 & 3 & 27 & 3 & 27 & 2 & 18 & 2 & 18 & 1 & 9 \\
\hline 14 & $\begin{array}{l}\text { Si me siento triste, } \\
\text { sé quién puede } \\
\text { animarme. }\end{array}$ & 1 & 9 & 0 & 0 & 0 & 0 & 0 & 0 & 2 & 18 & 8 & 73 \\
\hline 17 & $\begin{array}{l}\text { Me molesta que la } \\
\text { gente me pregunte } \\
\text { cómo estoy cuando } \\
\text { me encuentro mal } \\
\text { por algo que me } \\
\text { ha sucedido. }\end{array}$ & 2 & 18 & 4 & 36 & 3 & 27 & 0 & 0 & 2 & 18 & 0 & 0 \\
\hline 18 & $\begin{array}{l}\text { Me cuesta admitir } \\
\text { mi ignorancia } \\
\text { sobre un tema } \\
\text { cuando las personas } \\
\text { de mí alrededor me } \\
\text { piden opinión sobre } \\
\text { él. }\end{array}$ & 0 & 0 & 6 & 55 & 2 & 18 & 0 & 0 & 1 & 9 & 2 & 18 \\
\hline \multirow[t]{3}{*}{20} & \begin{tabular}{lr}
\multicolumn{3}{l}{ Me cuesta expresar } \\
mi alegría en \\
público \\
ejemplo \\
abrazos...)
\end{tabular} & 7 & 64 & 2 & 18 & 0 & 0 & 0 & 0 & 0 & 0 & 2 & 18 \\
\hline & $\begin{array}{l}\text { Puntajes globales } \\
\text { por opción }\end{array}$ & & & 29 & & 31 & & 45 & & 39 & & 33 & \\
\hline & $\begin{array}{l}\text { Media de la } \\
\text { percepción global } \\
\text { de la muestra }\end{array}$ & \multicolumn{4}{|c|}{ Ptmin. 6} & \multicolumn{4}{|c|}{ 20ptmax36 } & & & & \\
\hline
\end{tabular}

En el cuadro 4 se visualiza que la media de la percepción global de la muestra para la dimensión habilidad para expresar los sentimientos exhibe un resultado promedio de 20 
puntos, de dicho resultado se puede inferir según Bellés (2010) que los estudiantes encuestados exponen que poseen dificultad para expresar sus sentimiento en cuanto a la situación que acontece desde su cotidianidad, dichos resultado en referencia a lo planteado por la autora anterior permite señalar que dichos individuos tiene un grado de madurez medio y presentan poca seguridad en sus pensamientos y acciones.

Seguidamente, se muestra la tercera dimensión de este proyecto, referida a la sensibilidad:

Cuadro 5. Tabla referencial para la interpretación de la estabilidad emocional en la sensibilidad

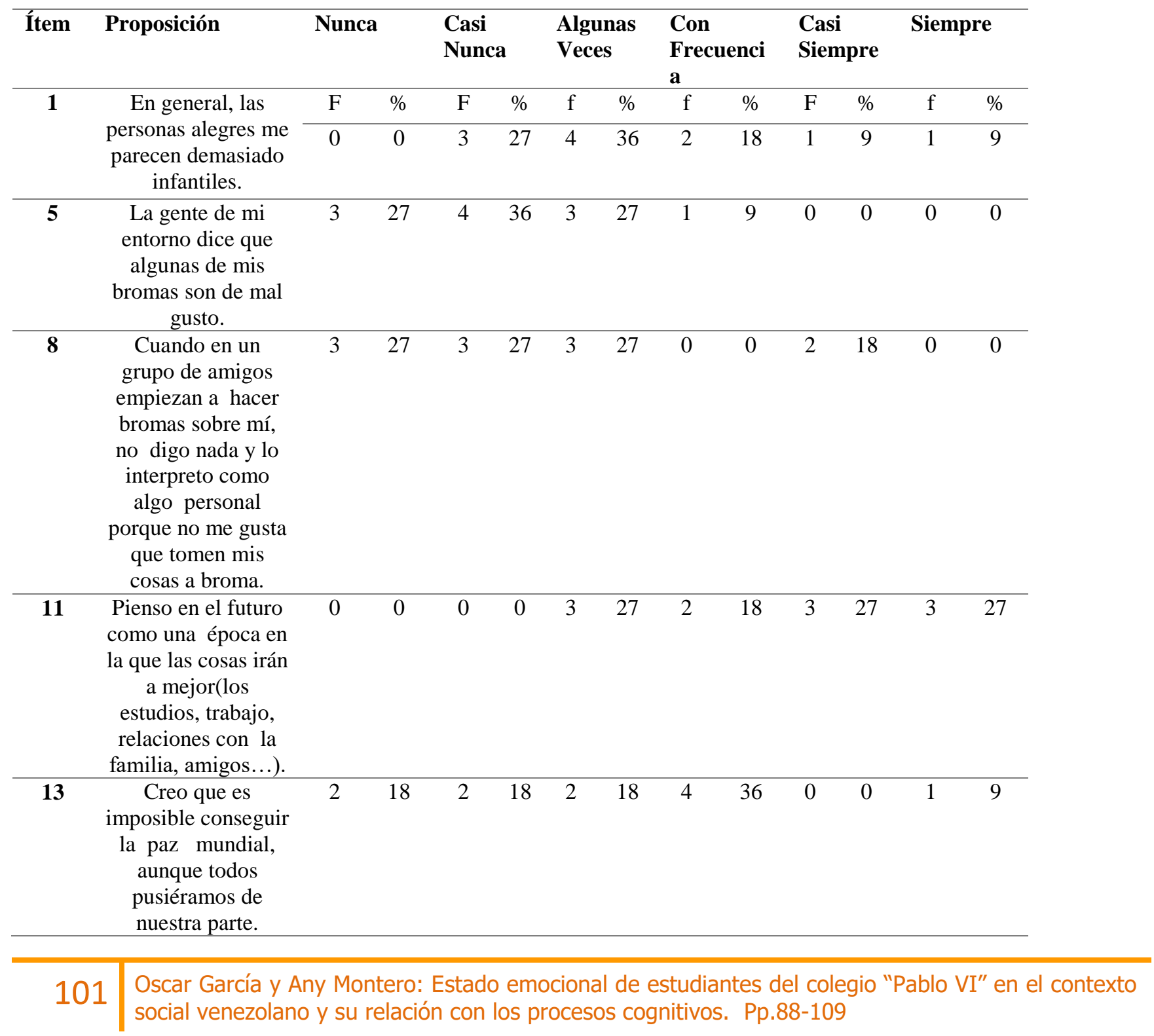




\begin{tabular}{|c|c|c|c|c|c|c|c|c|c|c|c|c|c|}
\hline 16 & $\begin{array}{l}\text { Cuando veo una } \\
\text { película me da } \\
\text { igual lo que le } \\
\text { pase al } \\
\text { protagonista, por } \\
\text { muy triste que sea. }\end{array}$ & 5 & 46 & 3 & 27 & 3 & 27 & 0 & 0 & 0 & 0 & 0 & 0 \\
\hline & $\begin{array}{l}\text { Puntajes globales } \\
\text { por opción }\end{array}$ & & 39 & & 34 & & 28 & & 50 & & 34 & & 20 \\
\hline & $\begin{array}{c}\text { Media de la } \\
\text { percepción global } \\
\text { de la muestra }\end{array}$ & \multicolumn{2}{|c|}{$\mathrm{Pt} \min 6$} & & & & & 19 & \multicolumn{3}{|c|}{ ptmax36 } & & \\
\hline
\end{tabular}

En el cuadro 5 se muestran los resultados del estudio para la dimensión sensibilidad por individuos y de forma global, se puede percibir que la media de la percepción global de la muestra para dicha dimensión exhibe un resultado promedio de 19 puntos, el mencionado valor puede interpretarse en las palabras de Bellés (2010) en la cual los estudiantes encuestados exponen un grado de sensibilidad intermedio, en tal sentido los mismos de forma general muestran una necesidad de afecto y comprensión ante las situaciones vividas en la cotidianidad y en el contexto social.

Finalmente, se encuentra la cuarta dimensión:

Cuadro 6. Tabla referencial para la interpretación de la estabilidad emocional en la deseabilidad social

\begin{tabular}{|c|c|c|c|c|c|c|c|c|c|c|c|c|c|}
\hline \multirow{2}{*}{$\begin{array}{l}\text { Ítems } \\
2\end{array}$} & \multirow{2}{*}{ 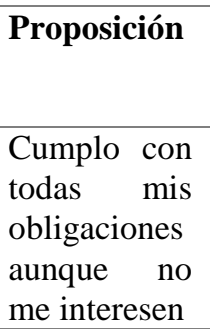 } & \multicolumn{2}{|c|}{ Nunca } & \multicolumn{2}{|c|}{$\begin{array}{l}\text { Casi } \\
\text { Nunca }\end{array}$} & \multicolumn{2}{|c|}{$\begin{array}{l}\text { Algunas } \\
\text { Veces }\end{array}$} & \multicolumn{2}{|c|}{$\begin{array}{l}\text { Con } \\
\text { Frecuencia }\end{array}$} & \multicolumn{2}{|c|}{$\begin{array}{l}\text { Casi } \\
\text { Siempre }\end{array}$} & \multicolumn{2}{|c|}{ Siempre } \\
\hline & & 0 & 0 & 0 & 0 & 2 & 18 & 2 & 18 & 4 & 36 & 3 & 27 \\
\hline 7 & $\begin{array}{l}\text { Realizo } \\
\text { todos los } \\
\text { favores que } \\
\text { me pide la } \\
\text { gente de mi } \\
\text { entorno. }\end{array}$ & 0 & 0 & 1 & 9 & 3 & 27 & 3 & 27 & 2 & 18 & 2 & 18 \\
\hline 12 & $\begin{array}{l}\text { Suelo } \\
\text { cotillear de } \\
\text { la gente a } \\
\text { sus espaldas. }\end{array}$ & 2 & 18 & 5 & 46 & 3 & 27 & 0 & 0 & 1 & 9 & 0 & 0 \\
\hline 21 & $\begin{array}{l}\text { Respeto } \\
\text { siempre la } \\
\text { opinión de } \\
\text { los otros en } \\
\text { el país }\end{array}$ & 0 & 0 & 2 & 18 & 2 & 18 & 1 & 9 & 4 & 36 & 2 & 18 \\
\hline
\end{tabular}




\begin{tabular}{|c|c|c|c|c|}
\hline $\begin{array}{l}\text { Puntajes } \\
\text { globales por } \\
\text { opción }\end{array}$ & 52 & 45 & 29 & 46 \\
\hline $\begin{array}{l}\text { Media de la } \\
\text { percepción } \\
\text { global de la } \\
\text { muestra }\end{array}$ & Ptmin 4 & & 16 ptmax 24 & \\
\hline
\end{tabular}

En el cuadro 6 se muestran los resultados del estudio para la dimensión deseabilidad social por individuos en estudio y de forma global, se puede entrever que la media de la percepción global de la muestra para dicha dimensión muestra un resultado promedio de 16 puntos con una máxima de 24 , dicho valor permite sustentar lo argumentado por las palabras de Bellés (2010) en la cual los estudiantes encuestados exponen un grado medio en cuanto a las expectativas de su contexto social, así como una acción media de iniciativa propia y la preocupación de la acción social.

En suma, se puede apreciar que los estudiantes encuestados presentan un grado de estabilidad moderada, en tal sentido esto permite visualizar con individuos medianamente serenos y que en oportunidades se sientes agobiados por los problemas y preocupaciones del entorno. Fernández-Abascal et.al. (2019). En tanto que es importante su atención ya que pueden presentar cambios bruscos de humor presentar ansiedad y tratar de evadir la realidad en las situaciones en cuales se les muestran conflictivas alterando de alguna forma la sincronía de su ser.

\section{Resultado del estudio correlacional entre la estabilidad emocional de la muestra y logro de los Procesos Cognitivos}

Una vez estudiadas las variables anteriores de forma separada con el propósito de describir y determinar el grado de estabilidad emocional de los individuos en el contexto social actual y logro o domino de los procesos cognitivos se procedió a establecer el grado de relación existente entre dichas variables. Para ellos se muestra un cuadro de los puntajes obtenidos por sujeto en cada uno de los test aplicados, obteniendo los siguientes resultados:

Cuadro 7. Relación de puntajes alcanzados por la muestra en los test de Estabilidad emocional y procesos cognitivos

Individuos Puntaje Test Estabilidad Puntaje Test Procesos




\begin{tabular}{ccc}
\hline & emocional (X) & cognitivos (Y) \\
\hline $\mathbf{1}$ & 67 & 12 \\
\hline $\mathbf{2}$ & 95 & 14 \\
\hline $\mathbf{3}$ & 77 & 12 \\
$\mathbf{4}$ & 68 & 8 \\
\hline $\mathbf{5}$ & 52 & 8 \\
\hline $\mathbf{6}$ & 65 & 8 \\
\hline $\mathbf{7}$ & 77 & 14 \\
\hline $\mathbf{8}$ & 68 & 4 \\
\hline $\mathbf{9}$ & 68 & 10 \\
\hline $\mathbf{1 0}$ & 65 & 10 \\
\hline $\mathbf{1 1}$ & 90 & 16 \\
\hline
\end{tabular}

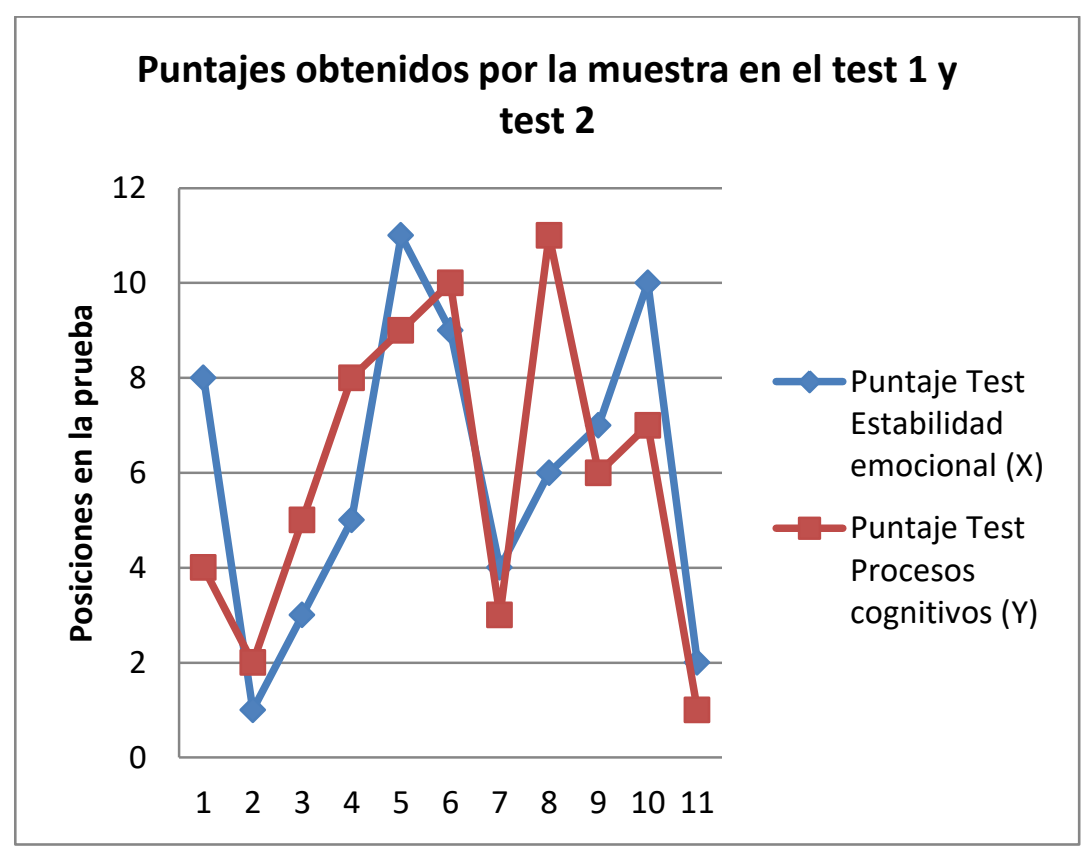

Gráfico 1. Relación de puntajes alcanzados por la muestra en los test de Estabilidad emocional y procesos cognitivos

Una vez determinados los puntajes alcanzados por la muestra se procedió al cálculo del grado de correlación existente entre las dos variables sometidas al estudio, debido a que las mismas son variables cuantitativas presentadas en una escala ordinal fue aplicado el coeficiente de correlación de Spearman (r), considerando para el presente caso la variable $\mathrm{x}=$ como el puntaje de la estabilidad emocional y la variable $\mathrm{Y}=$ al puntaje obtenido en logro de los procesos cognitivos.

Posterior a la aplicación del coeficiente de correlación, el resultado arrojado apunta que 
$\mathrm{r}=0.67$ para su interpretación fue utilizada la siguiente tabla consultada en el texto de Herrera y Montero, (2013):

Cuadro 8. Tabla de interpretación para la existencia de correlación.

\begin{tabular}{ll}
\hline \multicolumn{1}{c}{ Resultados } & \multicolumn{1}{c}{ Interpretación } \\
\hline 0 a 0.40 & Correlación Baja \\
\hline 0.41 a 0.60 & Correlación Moderada \\
\hline 0.61 a 0.90 & Correlación Alta \\
\hline 0.91 a 1,00 & Correlación perfecta \\
\hline
\end{tabular}

La derivación de los cálculos realizados permite establecer la existencia de una alta correlación positiva entre las dos variables sometidas al estudio. En tal sentido, se puede sostener que la estabilidad emocional del individuo que se encuentra en un contexto social inestable afecta consecuentemente el logro de los procesos cognitivos estudiados en la presente investigación, tal como lo señala Arranz (2017) al plantear, que los procesos cognitivos se pueden ver afectados por los estados emocionales de las personas ya que este participa o juega como un papel principal dentro de la motivación, desempeño y esfuerzo del individuo.

\section{Conclusiones}

Sobre la base de los objetivos de la presente investigación, las hipótesis planteadas y el análisis e interpretación de los resultados se llegó a las siguientes conclusiones:

1. Se evidenció que los estudiantes del primer año del colegio Pablo VI en su mayoría presenta un domino medio en de los procesos cognitivos, que tasa muy bajas se encuentran en los extremos del alcance de los objetivos propuestos exigido para su edad.

2. Asimismo, se pudo determinar que los estudiantes presentan un valor promedio general que exhibe una estabilidad emocional media lo cual conlleva que los estudiantes exhiban conductas emocionales de agobio, preocupación y poca madurez frente a la situación social, de tal manera que estos individuos se encuentran afectados y vulnerables frente a la realidad cotidiana en la cual viven.

3. En cuanto a las dimensiones ansiedad, habilidad de expresión de los sentimientos, sensibilidad y deseabilidad social los estudiantes muestran valores que tienden a estar en el centro de las tablas de interpretación lo que conlleva a visualizar que los estudiantes 
presentan algunas debilidades en el aspecto emocional o estado emocional.

4. El estudio permitió establecer la existencia de una correlación alta entre las variables sometidas a estudio como son el estado emocional percibido a través de la estabilidad emocional (Bellés, 2010) y el dominio de los procesos cognitivos. En tal sentido, existe una estrecha relación en cuanto a que el estudiante alcance el dominio de los procesos cognitivos y el estado emocional que el mismo presenta.

5. La situación económica y social del país se traduce en una variable interviniente que afecta el desarrollo o estabilidad emocional del individuo.

\section{Recomendaciones}

De las conclusiones obtenidas en el presente estudio se proponen las siguientes recomendaciones:

1. Difundir los resultados de esta investigación entre el personal docente, administrativo y directivo para que se busquen alternativas de enseñanza que ayuden a la mejora del estado emocional de los estudiantes.

2. Realizar talleres de actualización a los docentes relacionados con la elaboración de estrategias de enseñanzas basadas en inteligencia emocional.

3. Establecer jornadas con los padres para que conozcan el estado emocional de sus hijos y puedan buscar alternativas para la mejora del mismo.

4. Con la intención de profundizar en los resultados y conclusiones obtenidas, es altamente recomendable conducir la presente investigación bajo otros enfoques investigativos de naturaleza mixta y otros de naturaleza cualitativa, tomando en cuenta que desde estos enfoques pueden surgir resultados complementarios a los ya obtenidos, que pueden develar otras aristas de la realidad estudiada, que pudieran influir en el estado emocional el estudiante y en su proceso de aprendizaje.

\section{Referencias}

ABC de la Psicología (2012). Desarrollo cognoscitivo de los adolescentes. http://www.abc.com.py/articulos/desarrollo-cognoscitivo-de-los-adolescentes- 
693642.html. España.

Arias, F. (2006). El Proyecto de investigación. Introducción a la metodología científica. (5aed). Caracas: Episteme.

Arranz, A. (2017). Procesos cognitivos: Qué son y cómo podemos mejorar nuestros procesos mentales. Blogspot. https://blog.cognifit.com/es/procesos-cognitivos/

Bellés, M. (2010). Relación entre estilos de pensamiento y estrategias de evitación de los estudiantes de psicología. Jornadas de fomento de la Investigación. Universidad de Jaume.

Canelones, P. (2016). La crisis cambió el comportamiento de los venezolanos ¡La cruda realidad! Artículo en línea. https://resistenciav58.wordpress.com/2016/02/20/la-crisis-cambio-elcomportamiento-de-los-venezolanos-la-cruda-realidad-\%E2\%96\%BA/

Calvo, R. (2009). Cambios emocionales en los adolescentes, Revista Zona hospitalaria $\begin{array}{lllll}\text { medicina } & y & \text { salud. } & 1 & \text { (20). }\end{array}$ http://www.zonahospitalaria.com/V1/navarra/noticias/zh_20/cambios_emocionales. shtml. España.

Fernández-Abascal, E. Garcia, B., Jiménez, M., Martín, M, Domínguez, F. (2019). Psicología de la emoción. Editorial Universitaria Ramón Areces. Madrid. España.

Guía de Venezuela. (2017). Actualidad sobre Inseguridad en Venezuela. Disponible en, http://www.guia.com.ve/actualidad/inseguridad. Caracas.

Goleman, D. (1999). Inteligencia Emocional. Editorial Kairós. S.A. Barcelona. España.

Hernández, R; Fernández, C y Baptista, P. (2014). Metodología de la Investigación, Editorial McGraw-Hill, Interamericana de México. 6ta edición

Herrera, M.; Montero, A. (2013). Estadística a tu alcance, Editorial Fundación Buria. Caracas.

Hurtado I, Toro, J. (1998). Paradigmas y Métodos de Investigación en Tiempos de Cambio. Editorial CEC, S.A. Caracas, Venezuela.

Lozano, J. (2019). Las dificultades de aprendizaje en los centros educativos de enseñanza secundaria. Tesis Doctoral. Universidad Complutense de Madrid. Facultad de Educación. 
Muñoz, A. (2019). ¿Qué es la estabilidad emocional?.Blogspot. https://www.aboutespanol.com/que-es-la-estabilidad-emocional-396374

Ocaña, A. (2015). La concepción de Maturana acerca de la conducta y el lenguaje humano. $\begin{array}{lllll}\text { CES Psicología, } & 8, & \text { (2), } & \text { pp. }\end{array}$ http://www.redalyc.org/articulo.oa?id=423542417011.

Pérez, C. (2012). El bloqueo emocional. https://lamenteesmaravillosa.com/bloqueoemocional/. Argentina.

Porto, J. (2011). Inteligencia artificial. https://definicion.de/inteligencia-artificial/. Colombia.

Revilla, M (2010). América Latina y los movimientos sociales: el presente de la «rebelión del coro». Nueva Sociedad. 22. https://nuso.org/articulo/america-latina-y-losmovimientos-sociales-el-presente-de-la-rebelion-del-coro/

Rodríguez, G.,Gil, J., García, E. (1999). Metodología de la investigación cuantitativa. Málaga, España: Ediciones ALJIBE.

Ruíz Bolívar, C. (2013). Un enfoque cuantitativo y cualitativo para la recolección y análisis de datos. Libro en línea. Danaga Trading and consulting. 3era edición. https://www.academia.edu/37886948/Instrumentos_y_Tecnicas_de_Investigaci\%C 3\%B3n_Educativa_Carlos_Ruiz_Bolivar_pdf.

Sabino, C. (2002). Como Hacer una Tesis. Editorial Panapo, 3da. Edición, Caracas, Venezuela.

Tamara, A. (2016). Reacciones emocionales e intervención psicológica en niños. Documento en línea .http://intellectum.unisabana.edu.co/bitstream/handle/10818/4530/131016.pdf?sequ ence $=1$. [Consulta Marzo, 2017].

Tamayo, M. (2000). El proceso de la investigación científica. México: Limusa Noriega Editores.

Viera, S. (2014). Estado emocional de niños, Estudio de caso. España.

Villalta, M. Assael, C., Martinic, S. (2012). Conocimiento escolar y procesos cognitivos en la interacción didáctica en la sala de clase. Educativos 35(141):84-96. DOI: 10.1016/S0185-2698(13)71836-1 